\title{
Quantum Electron-Phonon Interaction for Transport in Open Nanostructures
}

\author{
P. Bordone, R. Brunetti, M. Pascoli, C. Jacoboni \\ Istituto Nazionale per la Fisica della Materia, Dipartimento di Fisica \\ Via Campi 213/A, I-41100 Modena, Italy
}

\begin{abstract}
Electronic quantum transport acounting for coherent propagation and electronphonon (e-p) scattering has been used to calculate the current-voltage characteristic for model nanostructures.
\end{abstract}

\section{Introduction}

Transport in mesoscopic systems has been widely investigated in recent years [1] due to the fundamental physics involved and the possible application to electron-device modelling. Since the dimensions of mesoscopic systems are comparable with typical electron coherence lengths, a correct analysis of transport phenomena in such systems requires a detailed quantum-mechanical treatment. The theoretical approach based on the concept of Wigner function (WF), is a phase space formulation of quantum mechanics that allows to establish many analogies with the semiclassical theory based on the concept of distribution function. From the knowledge of the WF all physical information relative to the transport process can be extracted. In our case the Wigner evolution equation has been solved by using the perturbation theory as it regards the phonon scattering, while the external fields are incorporated into the unperturbed Hamiltonian.

\section{Theoretical approach}

A WF for the coupled system of electrons and phonons has been recently introduced [2], which provides the electron WF once the trace over the phonon variables is performed. An integral equation for this function can be written [3] and applied to study quantum electron transport in presence of scattering in an open system occupaying a finite region inside given boundaries [4]. The problem is not trivial owing to the nonlocality of the interaction and to the presence of integrations over the space coordinates into the general equation for the WF. We have proved [5] that for the solution of the integral equation mentioned above it is possible (as for the semiclassical Boltzmann equation) to substitute the knowledge of the WF over all space at a given (initial) time $t_{\circ}$ with the knowledge of the same function inside the region of interest at $t_{\circ}$ and at all times at the boundary for "entering" momenta. In other words: a solution is obtained such that the scattering with phonons inside the 
device does not alter the boundary condition for the WF. The use of this property allows to incorporate into the model available information about conditions imposed at the boundaries of real devices.

The integral equation for such a situation results to be:

$$
\begin{aligned}
& f_{w}\left(z, p_{z}, n_{q}, n_{q}^{\prime}, t\right)= \\
& \frac{1}{h} \sum_{n n^{\prime}} f_{n n^{\prime}}\left(z, p_{z}\right) e^{-i\left(\omega\left(n n_{q}\right)-\omega\left(n^{\prime} n_{q}^{\prime}\right)\right)\left(t-t_{o}\right)} \int_{-A}^{A} d z^{\prime} \int d p_{z}^{\prime} f_{n n^{\prime}}^{*}\left(z^{\prime}, p_{z}^{\prime}\right) f_{w}\left(z^{\prime}, p_{z}^{\prime}, n_{q}, n_{q}^{\prime}, t_{\circ}\right)+ \\
& \frac{1}{h} \sum_{n n^{\prime}} f_{n n^{\prime}}\left(z, p_{z}\right) \int_{t_{0}}^{t} d t^{\prime} e^{-i\left(\omega\left(n n_{q}\right)-\omega\left(n^{\prime} n_{q}^{\prime}\right)\right)\left(t-t^{\prime}\right)}\left\{\left[\int_{0}^{+\infty} d p_{z}^{\prime} \frac{p_{z}^{\prime}}{m} f_{n n^{\prime}}^{*}\left(-A, p_{z}^{\prime}\right) \times\right.\right. \\
& \left.f_{w}\left(-A, p_{z}^{\prime}, n_{q}, n_{q}^{\prime}, t^{\prime}\right)-\int_{-\infty}^{0} d p_{z}^{\prime} \frac{p_{z}^{\prime}}{m} f_{n n^{\prime}}^{*}\left(A, p_{z}^{\prime}\right) f_{w}\left(A, p_{z}^{\prime}, n_{q}, n_{q}^{\prime}, t^{\prime}\right)\right]+ \\
& \int_{-A}^{A} d z^{\prime} \int d p_{z}^{\prime} \sum_{m m_{q}}\left[\mathcal{H}^{\prime}\left(n n_{q}, m m_{q}\right) f_{m n^{\prime}}^{*}\left(z^{\prime}, p_{z}^{\prime}\right) f_{w}\left(z^{\prime}, p_{z}^{\prime}, m_{q}, n_{q}^{\prime}, t^{\prime}\right)-\right. \\
& \left.\left.f_{n m}^{*}\left(z^{\prime}, p_{z}^{\prime}\right) f_{w}\left(z^{\prime}, p_{z}^{\prime}, n_{q}, m_{q}, t^{\prime}\right) \mathcal{H}^{\prime}\left(m m_{q}, n^{\prime} n_{q}^{\prime}\right)\right]\right\} .
\end{aligned}
$$

where for simplicity a one-dimensional case has been considered. In the previous equation $\mathcal{H}^{\prime}$ contains the scattering agents (in our case e-p polar-optical interaction), $\hbar \omega\left(n, n_{q}\right)$ indicates the total unperturbed energy of the e-p system when the electron is in the $\mathrm{n}$-th eigenstate and the phonon bath is in a state with $n_{q}$ phonons in mode $\mathrm{q}$, and $\mathcal{H}^{\prime}\left(n n_{q}, m m_{q}\right)$ are its matrix elements on the basis $\left\{\left|n, n_{q}\right\rangle\right\}$ of the unperturbed Hamiltonian. Furthermore the coefficients $f_{l m}(\mathbf{r}, \mathbf{p})$ are given by

$$
f_{l m}(\mathbf{r}, \mathbf{p})=\int d \mathbf{r}^{\prime} e^{-i \frac{\mathrm{R}}{h} \mathbf{r}^{\prime}}\left\langle\mathbf{r}+\frac{\mathbf{r}^{\prime}}{2} \mid l\right\rangle\left\langle m \mid \mathbf{r}-\frac{\mathbf{r}^{\prime}}{2}\right\rangle
$$

Finally $-A$ and $A$ are the device boundaries. It has been shown that a Monte Carlo solution allows for a proper average over the phonon coordinates, as long as hotphonon effects are neglected [6].

We have applied a numerical procedure to evaluate the itcrative expansion of Eq. (1) up to the second order in the e-p perturbation Hamiltonian. Integrations over time variables have been performed analytically; the integrals over $z^{\prime}, p^{\prime}$ and over the electron states have been performed numerically, while the integral over the phonon modes has been performed by means of a Monte Carlo sampling. Space correlations for the evaluation of the coefficients in Eq. (2) have been considered up to $40 \mathrm{~nm}$ [4].

\section{Results}

The theory presented above has been applied to calculate the electron current across a potential step and a double barrier under the hypotesis that the device is so small that electrons experience at most one scattering event while crossing the active region.

Fig. 1 shows the current as a function of the step potential height as obtained with the ballistic WF (solid curve) and with the WF corrected by the effect of an e-p scattering process (circles) switched on 50 fs before the "observation time". Comparison is presented with the outcome of a semiclassical calculation based on the Boltzmann equation. The quantum ballistic curve is always lower then the corresponding semiclassical one, and instead of reaching a saturation value, it rises up to a maximum and then decreases. This is due to the fact that in the quantum picture increasing 


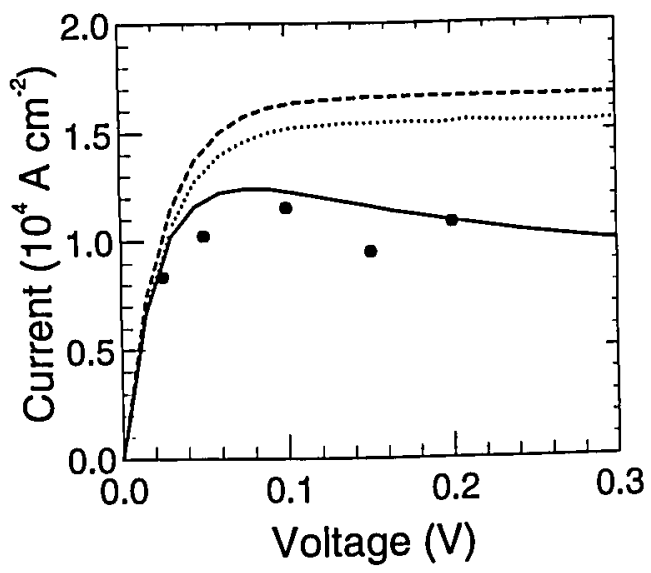

Figure 1: Current-voltage characteristic for electrons crossing a step potential, (see text).

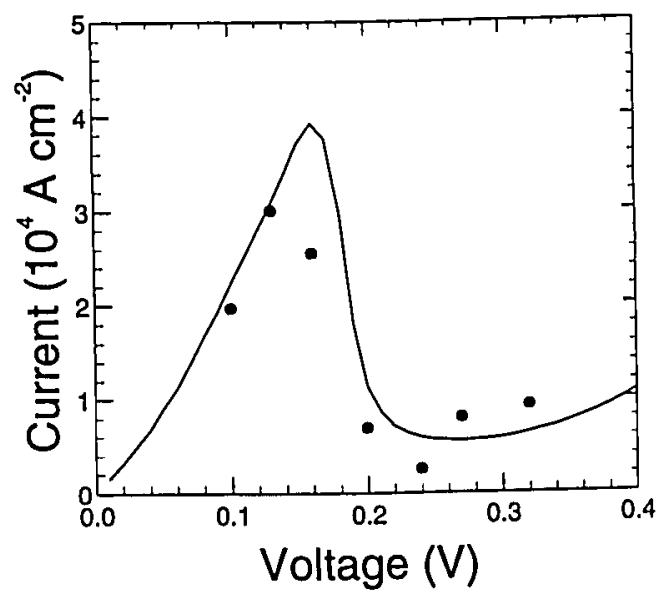

Figure 2: Current-voltage characteristic for clectrons crossing a resonant-tunneling diode (see text).

the potential step leads, as a consequence of quantum reflection, to a decrease of the transmission coefficient, that is, to a decrease of the current intensity. Finally, in both quantum and semiclassical calculations the effect of the e-p interaction is, as expected, a reduction of the current with respect to the ballistic case. Fig. 2 shows the current voltage characteristic of a double-barrier resonant-tunneling diode. The two barriers are $0.28 \mathrm{eV}$ high and $2.8 \mathrm{~nm}$ wide, and their distance is $5 \mathrm{~nm}$. The solid curve is obained with the ballistic WF, while the full circles are results of the calculations performed using the WF corrected by the effect of an e-p scattering process, switched 
on $50 \mathrm{fs}$ before the "observation time". The inclusion of the e-p interaction reduces the current peak/valley ratio, in agreement with the results obtained with simpler models $[7,8]$.

\section{References}

[1] See, e.g.: Quantum Transport in Ultrasmall Devices, edited by D. K. Ferry, H. L. Grubin, C. Jacoboni and A. P. Jauho, Plenum Press, New York, 1994; S. Datta: Electronic Transport in Mesoscopic Systems, Cambridge Press, Cambridge (1995).

[2] F. Rossi and C. Jacoboni and M. Nedjalkov, "A Monte Carlo solution of the Wigner transport equation," Semicond. Sci. Technol., vol. 9, pp. 934-936, 1994.

[3] C. Jacoboni, A. Abramo, P. Bordone,R. Brunetti, and M. Pascoli, "Application of the Wigner-Function Formulation to Mesoscopic Systems in Presence of ElectronPhonon Interaction," VLSI Design to appear.

[4] P. Bordone, A. Abramo, R. Brunetti, M. Pascoli, and C. Jacoboni, "Wigner Function for OPen Systems with Electron-Phonon Interaction," Phys. stat. sol. (b), vol. 204, pp. 303-305, 1997.

[5] A. Abramo, P. Bordone, R. Brunetti, C. Jacoboni, and M. Pascoli "Quantum transport of electrons in open nanostructures with the Wigner-function formalism," to be published

[6] R. Brunetti and C. Jacoboni and F. Rossi, "Quantum theory of transient transport in semiconductors: A Monte Carlo Approach," Plys. Rev. B, vol. 31, pp. 1078110790, 1989.

[7] W. R. Frensley, "Quantum transport modelling of resonant-tunneling devices" Solid-State Electron, vol. 31, pp. 739-742, 1988.

[8] S. Ragazzi, A. Di Carlo, P. Lugli, and F. Rossi, "Analysis of Quantum-Transpot Phenomena in Mesoscopic Systems: A Monte Carlo Approach," Pliys. stat. sol. (b), vol. 204, pp. 339-342, 1997. . 